\title{
THE MOTT TRANSITION IN DISORDERED SYSTEMS
}

\section{Dobrosavljević and G. Kotliar}

Serin Physics Laboratory, Rutgers University, Piscataway, NJ 08854, USA

We review the recent progress in understanding the Mott transition in disordered systems using the mean-field approach to the strong correlation problem. It is based on a new functional integral formulation of the disordered interacting electron problem. The saddle-point solution recovers a mean-field theory of the strong correlation problem, and becomes exact in the limit of large spatial coordination. We compare the results of the mean-field theory with experimental findings and indicate directions for future work.

PACS numbers: $75.20 . \mathrm{Hr}, 71.55$.Jv

\section{Introduction}

The Mott transition, that is the metal-insulator transition induced by the electron-electron interactions in a periodic system, has been investigated theoretically and experimentally for many years [1]. Experimentally, it seems to be realized in three-dimensional transition metal oxides such as $\mathrm{V}_{2} \mathrm{O}_{3}$ [2] and can be driven by varying pressure, temperature, and composition.

From a theoretical point of view, several ideas have been put forward as a pictures of the Mott transition. In early work, Hubbard introduced the notion of Hubbard bands [3], which are electronic bands describing propagating empty and doubly occupied sites. For large $U$ these bands split, leading to an insulating state. As $U$ is reduced, there is a critical value of $U$ where the two bands merge, and the system becomes metallic. This is the Hubbard picture of the metal-insulator transition. An alternative picture was provided by Brinkman and Rice [4], building on the work of Gutzwiller. These authors started from the metallic phase, which they described as a strongly renormalized Fermi liquid with a characteristic Fermi energy scale $\epsilon_{\mathrm{F}}^{*}$, gradually collapsing as the transition is approached. The metal-insulator transition in this view is driven by the disappearance of the Fermi liquid quasiparticles. Another scenario was proposed by Slater [5], who pointed out that the metal-insulator transition is often accompanied by long range antiferromagnetic order, and viewed the doubling of the unit cell which makes the band structure of the system that of a band-insulator, as the driving force behind the metal-insulator transition.

The nature of the Mott transition in periodic solids has been recently clarified by the solution of a mean-field theory which becomes exact in the limit of large spatial coordination [6]. The approach is based on a mapping of the models of strongly 
correlated electrons onto impurity models supplemented by a self-consistent condition [7]. This approach provided a synthesis of the Mott Hubbard Brinkman Rice and Slater pictures, and many predictions of the approach agree qualitatively and even quantitatively with experimental results in yarious transition metal oxides.

A second possible experimental realization of the Mott transition is provided by phosphorus doped silicon (Si:P) and other uncompensated doped semiconductors [11]. Here the metal-insulator transition can be driven by changing the doping or by applying a magnetic field. Randomness introduçes a new important variable in this problem, raising various new questions. One can ask how disorder modifies various regimes of a strongly correlated metal, or more specifically, how weak disorder affects a strongly correlated metal and a Mott insulating phase. In addition, even for weak or zero interactions, disorder alone can give rise to a metal-insulator transition (Anderson transition), and to an Anderson insulating phase [12].

Most of the theoretical work on the metal-insulator transition induced by disorder and interaction begins with the Anderson transition of the non-interacting system and adds the interactions perturbatively. The perturbation theory is then controlled by a renormalization group near two dimensions, an approach which was pioneered by Finkelshtein [13]. This approach, based on Fermi liquid ideas, has provided a consistent framework for understanding the metal-insulator transition in systems where spin is not conserved. In systems where spin is conserved, this approach suggests that at long scales the interactions are strong while the disorder is weak [8] but the formalism breaks down at that point and no consistent interpretation exists.

This situation has motivated us to extend the mean-field approach to the disordered interacting systems $[9,10,14]$. We approach the metal-insulator transition from the limit of large dimensionality (as opposed to the epsilon expansion around two dimensions). In this limit; the physics is dominated by the strong interactions in a disorder medium rather than by Anderson localization. We regard the metal-insulator transition in. uncompensated doped semiconductors as a genuine Mott transition and explore the consequences of this view using a recently developed mean-field theory of strongly correlated electron systems. The absence of long range magnetic ordering in Si:P and many other experiments suggest that this system displays a genuine Mott transition, making an interesting system to study the metal-insulator transition.

In this paper, we will review the main insights obtained with this approach. We stress a technical advantage of dealing with a spatially disordered situation, the possibility of carrying out a controlled loop expansion. We compare our results with the experimental situation in doped semiconductors and indicate directions for future work.

\section{Model of disorder}

We consider the following disordered Hubbard model Hamiltonian:

$$
H=\sum_{i j} \sum_{\sigma}\left(-t_{i j}+\varepsilon_{i} \delta_{i j}\right) c_{i, \sigma}^{\dagger} c_{j, \sigma}+U \sum_{i} c_{i, \uparrow}^{\dagger} c_{i, \uparrow} c_{i, \downarrow}^{\dagger} c_{i, \downarrow} \text {. }
$$

For simplicity, we restrict our attention to the class of models that can be 
formulated on an arbitrary lattice, but correspond to a special form of disorder. In this class, the random hopping elements are assumed to take the form

$$
t_{i j}=y_{i j} g\left(x_{i}, x_{j}\right)
$$

and in addition, there can be an arbitrary distribution of site energies $\varepsilon_{i}$. Here, $y_{i j}$-s are independent bond variables with a symmetric distribution, i.e. $\overline{y_{i j}^{2 n+1}}=0$, and $g\left(x_{i}, x_{j}\right)$ is an arbitrary function of local site variables $x_{i}$.

The special class of models which have a symmetric distribution of hopping elements has a very simple physical interpretation. As first observed by Wegner [15], in these "gauge invariant" models, the phases of the electrons undergo random shifts at every lattice hop and so the mean free path $\ell$ reduces to one lattice spacing. On general grounds, on length scales longer than $\ell$ the details of the lattice structure are washed out by disorder, so that for gauge invariant models in the large dimensionality limit, the details of the lattice structure become irrelevant. We contrast this with the models with arbitrary disorder, discussed in Sec. 3.2, which have a well-defined pure limit, and accordingly can also have an arbitrarily large mean free path. The presence of this intermediate lengthscale $(\ell$ can be much larger than the lattice spacing $a$, but much smaller than the localization length $\xi$ ) is often irrelevant to both the long wavelength phenomena such as localization, and local phenomena such as the Mott transition. The gauge invariant models avoid these unnecessary complications, without disrupting any of the qualitative properties on either very short, or very long lengthscales.

We will restrict our attention to the situation where $g\left(x_{i}, x_{j}\right)=x_{i} x_{j}$, with an arbitrary distribution $P_{X}\left(x_{i}\right)$ for the site variables $x_{i}$. For a trivial choice of $P_{X}\left(x_{i}\right)=\delta\left(x_{i}-1\right)$, the models reduce to the gauge invariant models of Wegner [15]. Nontrivial distributions $P_{X}\left(x_{i}\right)$ which extend to small values of the variable $x_{i}$ are useful for the study of disorder-induced local moment formation. Those sites with $x_{i}$ small represent the sites with weak hybridization. At intermediate correlation, we expect the sites with $x_{i}$ small to behave as local moments and give large contributions to the thermodynamic quantities such as the specific heat coefficient $\gamma=C / T$, while other sites remain in the itinerant regime.

Also, we take $y_{i j}$-s to be Gaussian random variables with zero mean, and with the variance

$$
\overline{y_{i j}^{2}}=\frac{1}{z} f_{i j} t^{2}
$$

Here, the (uniform) matrix $f_{i j}$ specifies the lattice structure

$$
f_{i j}= \begin{cases}1, & \text { for connected sites } \\ 0, & \text { for disconnected sites }\end{cases}
$$

and we have scaled the (square of the) hopping elements by the coordination number $z=\sum_{j} f_{i j}$, in order to obtain a finite result in the $z \rightarrow \infty$ limit.

In the $d=\infty$ limit, all local correlation functions can be evaluated with respect to a local effective Action of the form

$$
\begin{aligned}
S_{\mathrm{eff}}(i) & =\sum_{\sigma} \int_{0}^{\beta} \mathrm{d} \tau \int_{0}^{\beta} \mathrm{d} \tau^{\prime} c_{i, \sigma}^{\dagger}(\tau)\left[\delta\left(\tau-\tau^{\prime}\right)\left(\partial_{\tau}+\varepsilon_{i}-\mu\right)+W_{i, \sigma}\left(\tau, \tau^{\prime}\right)\right] c_{i, \sigma}\left(\tau^{\prime}\right) \\
& +U \int_{0}^{\beta} \mathrm{d} \tau c_{i, \uparrow}^{\dagger}(\tau) c_{i, \uparrow}(\tau) c_{i, \downarrow}^{\dagger}(\tau) c_{i, \downarrow}(\tau) .
\end{aligned}
$$


Here, the Fermi fields $c_{i, \sigma}(\tau)$ represent electrons of spin $\sigma$ on site $i, \mu$ is the chemical potential, and $\beta$ is the inverse temperature. The "Weiss field" $W_{i, \sigma}\left(\tau, \tau^{\prime}\right)$ is obtained by integrating out formally all the degrees of freedom on other sites in the lattice, in the large $d$ limit. The self-consistency condition determining $W_{i, \sigma}$ is

$$
W_{i, \sigma}\left(\omega_{n}\right)=\sum_{j} t_{i j}^{2} G_{j, \sigma}\left(\omega_{n}\right) \stackrel{d \rightarrow \infty}{\longrightarrow} \overline{t_{i j}^{2} G_{j, \sigma}\left(\omega_{n}\right)},
$$

where $G_{j, \sigma}\left(\omega_{n}\right)=\left\langle c_{j, \sigma}^{\dagger}\left(\omega_{n}\right) c_{j, \sigma}\left(\omega_{n}\right)\right\rangle$ are local Green's functions evaluated with respect to the effective action of $\mathrm{Eq}$. (2).

The present equations are exact at $z \rightarrow \infty$ for an arbitrary lattice, due to the presence of the "gauge invariant" form of the hopping disorder. We mention that the same equations are also exact on a $z=\infty$ Bethe lattice with an arbitrary form of disorder.

It is worth pointing out that there are two classes of lattices which can have large coordinations:

(a) Lattices with short-range bonds but living in a space of large dimensionality. For example, on a hypercubic lattice with nearest neighbor hopping in $d$ dimensions, $z=2 d$.

(b) Lattices embedded in a finite dimensional space, but having long hopping range. In this case, the lattice matrix $f_{i j}$ takes the form

$$
f_{i j}= \begin{cases}1, & ] i-j]<L, \\ 0, & \text { otherwise }\end{cases}
$$

and the coordination number $z \sim L^{d}$.

We emphasize the fact that for gauge invariant models of disorder the equations are independent of the lattice structure. Since the equations determine the local effective action, this means that all the local correlations functions will be insensitive to the lattice structure, in this mean-field limit. However, other properties such as the tendency to the formation of the spin and charge density wave, are very sensitive to the details of the lattice structure. In doped semiconductors [11], disorder introduces large amounts of frustration, and magnetic ordering does not occur, even though the system is strongly correlated, therefore we will concentrate on paramagnetic solutions of the mean-field equations which are realized on frustrated lattices.

\section{Thermodynamic and transport properties}

Once local Green's functions are self-consistently determined, we can immediately obtain all the thermodynamic properties from the calculation of the energy $E(T)$. Using standard methods [16], it is possible to express the energy in terms of single-particle Green's functions. In the following, we will concentrate on gauge invariant models, for which the expression takes, are particularly simple.' The energy of the system takes the form

$$
E(T)=\int \mathrm{d} \varepsilon_{i} P_{S}\left(\varepsilon_{i}\right) \int \mathrm{d} x_{i} P_{H}\left(x_{i}\right) \frac{1}{\beta} \sum_{n}\left[i \omega_{n}+\mu+W_{i}\left(\omega_{n}\right)\right] G_{i}\left(\omega_{n}\right) .
$$

The expression is valid in the paramagnetic phase, where we have performed the spin sum. It is important to note that the energy takes an additive form with 
respect to sites having energies $\varepsilon_{i}$ and hopping parameters $x_{i}$. Once the energy is known as a function of temperature, we can calculate quantities such as the specific heat, entropy, etc. We immediately conclude that the specific heat is also additive, reminiscent to the phenomenological "two-fluid model" of doped semiconductors [11]; we will discuss the relevance of our results to such systems in more details in Sec. 6.

The transport coefficients, like the conductivity can be obtained from Kubo formulas which reduce to calculating appropriate electronic correlation functions. In the case of the conductivity, the calculations are especially simple in the $d=\infty$ limit, essentially due to the cancellation of the vertex corrections, as first pointed out by Khurana [17]. For general models of disorder, it is then possible to express the conductivity in terms of the (averaged) local spectral functions - a feature reminiscent of the coherent potential approximation (CPA). In fact, in the non-interacting limit, our self-consistency conditions reduce to CPA, which is consistent with the absence of Anderson localization in $d=\infty$.

The considered class of gauge invariant models is characterized by hopping elements with random signs, which corresponds to a mean free path of one lattice spacing. The details of the lattice structure-become irrelevant, and the real part of the conductivity assumes a simple form

$$
\sigma_{\mathrm{R}}(\omega)=4 \pi a^{2} t^{2} \int \mathrm{d} \omega^{\prime} \rho_{W}\left(\omega^{\prime}\right) \rho_{W}\left(\omega^{\prime}+\omega\right) \frac{f\left(\omega^{\prime}\right)-f\left(\omega^{\prime}+\omega\right)}{\omega},
$$

where $f(\omega)$ is the Fermi function, and $\rho_{W}(\omega)$ is the local spectral function corresponding to the averaged cavity field

$$
\rho_{W}(\omega)=-\frac{1}{\pi} \operatorname{Im} \int \mathrm{d} \varepsilon_{i} P_{S}\left(\varepsilon_{i}\right) \int \mathrm{d} x_{i} P_{H}\left(x_{i}\right) x_{i}^{2} G_{i}(\omega+\mathrm{i} \eta) .
$$

At $\omega=0$, and low temperatures, the expression reduces to

$$
\sigma_{\mathrm{DC}}(T)=4 \pi a^{2} t^{2} \rho_{W}^{2}(\omega=0, T) .
$$

As we can see from this expression, the conductivity is finite in the metallic region $\left(\rho^{2}(\omega=0, T) \neq 0\right)$, in contrast to the case of pure lattices where the resistance (inelastic scattering) vanishes at $T \rightarrow 0$. For gauge invariant models, the pure limit cannot be obtained by tuning a parameter (disordered strength) since the mean free path $\ell$ cannot exceed one lattice spacing ( $\ell \rightarrow \infty$ in the pure limit). However, as we will see, these models with hopping randomness (in addition to possible site randomness) display generic behavior at the metal-insulator transition, which presumably is relevant for realistic systems.

\section{Metal-insulator transitions}

In general, when either sufficiently strong correlations or disorder is present, metal to insulator transitions (MIT) can take place. In the $d=\infty$ framework, the Anderson localization effects are absent, but MIT can still take place because of strong correlation effects. In the following, we discuss the possible classes of MIT-s present in $d=\infty$ models, and make predictions about the behavior of thermodynamic and transport properties in the vicinity as the transition is approached. 


\subsection{Hopping disorder}

In a number of materials that display a metal-insulator transition such as uncompensated doped semiconductors [11], the main source of disorder stems from random position of dopant atoms, thus leading to strong hopping randomness. In order to study such situations, we begin our analysis by examining the models with pure hopping randomness.

As the first example, we consider the case of simple uncorrelated hopping disorder, of the form $t_{i j}=y_{i j}$, with the $y_{i j}$ Gaussian distributed random numbers and the variance $t^{2}$. In that case, the self-consistency conditions determining the effective action are identical as for the pure Hubbard model on the $d=\infty$ Bethe lattice; the solution of this model is well known [18]. At moderate correlation strength, the system is metallic, and displays usual Fermi liquid behavior. At $T=0$, a Mott-Hubbard transition takes place at a critical interaction strength $U_{c}$, where the quasiparticle band vanishes and a gap for charged excitations appears, leading to an insulating state. As the transition is approached, the effective mass, and thus also the specific heat coefficient $\gamma=C / T$ is found to diverge as $\gamma \sim$ $\left(U_{c}-U\right)^{-1}$.

While these properties are identical as in the pure model, the behavior of the conductivity proves to be more sensitive to the presence of disorder. By using the expressions for the conductivity of Sec. 3, and the well-known results for the local spectral functions, we can readily compute the transport properties at arbitrary temperature $T$ and interaction strength $U$. At $T=0$ the behavior of the DC $(\omega=0)$ conductivity is particularly simple, and can be computed analytically. In this limit, Fermi liquid theorems for the Anderson model assert that in the metallic phase, the imaginary part of the Anderson model self energy vanishes physically this reflect absence of inelastic scattering at $T=0$. When this result is applied to the $d=\infty$ model, we conclude that at half-filling (at particle-hole symmetry), the local spectral function $\rho(\omega=0, T=0)$ is pinned to its non-interacting value $\rho_{0}=(\pi t)^{-1}$. As a result the $T=0$ value of the DC conductivity is also pinned, i.e. $\sigma_{\mathrm{DC}}(T=0)=\sigma_{0}=4 a^{2} / \pi$,

throughout the metallic phase. Although the precise value of $\sigma_{0}$ given here is appropriate only for this particular model of hopping randomness, the proof for the conductivity pinning can be easily generalized to more complicated situations. In fact, pinning is obeyed at $T=0$ for any model obeying (local) particle-hole symmetry, i.e. models at half-filling with an arbitrary form of hopping randomness. Random site energies break local particle-hole symmetry, and thus violate the pinning condition; in that case $\sigma_{\mathrm{DC}}(T=0)$ can depend on $U$.

Thus, we have shown that for models with hopping randomness, the conductivity at $T=0$ remains constant inside the metallic phase, and than abruptly jumps to zero as the system becomes a Mott insulator. The behavior can be described as a manifestation of minimum metallic conductivity, in agreement with early ideas of Mott [1].

At finite temperature, the pinning condition is violated due to inelastic scattering, and we have to solve explicitly the self-consistency equations at $T \neq 0$ in order to obtain results for the conductivity. Using methods of Ref. [10, 14], we have carried out the appropriate calculations, and the results are presented in Fig. 1. 


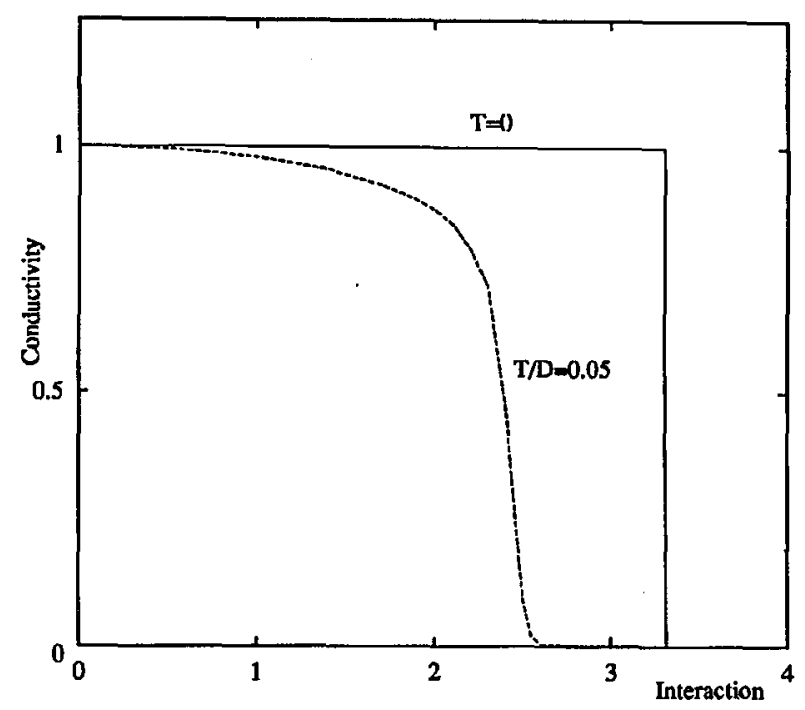

Fig. 1. DC conductivity of the infinite dimensional Hubbard model with random hopping at half-filling. $\sigma_{\mathrm{DC}}$ is plotted in units of $\sigma_{0}$, the $U=0$ value. The full line shows the $T=0$ behavior, displaying minimum metallic conductivity. At finite temperature $T=0.05>T^{*}$ (in units of the half-bandwidth $D=2 t$ ), $\sigma_{\mathrm{DC}}$ continuously decreases as the correlations grow, as shown by the dashed line.

The jump in the conductivity persists at small but finite temperatures $T<T^{*}$, which is of the order of one percent of the bandwidth. At higher temperatures, the conductivity is a smooth function of $U$, and continuously drops to exponentially small values as $U$ is increased, reflecting the destruction of the coherent quasiparticles by thermal inelastic scattering.

Before leaving the models with pure hopping disorder, we would like to comment on possible modifications of the transition in presence of more complicated models of random hopping. The generalizations, that we have in mind, correspond to correlated hopping disorder, where in addition to random bond variables $y_{i j}$, the hopping elements can take a more general form $t_{i j}=y_{i j} x_{i} x_{j}$, introduced in Sec. 2. For these models, the behavior of the system proves to be very sensitive to the detailed form of the probability distribution for random variables $x_{i}$. If the low- $x$ tail of the distribution extends to very small values, local moment formation occurs, and even the qualitative nature of the metallic phase can be modified; this effect will be discussed in more detail in Sec. 5. On the other hand, it is the form of the high-x tail that is the most relevant for the metal-insulator transitions. If this tail extends to very high $x$ values, it can suppress the Mott transition to arbitrarily high values of $U_{\mathrm{c}}$, since the band tails tend to close the Mott gap. The presence of long but finite tails leads only to quantitative modifications; in the case of algebraically long tails, the Mott (insulating) phase can be completely suppressed in the strict $d=\infty$ limit. In that case the sharp transition turns into a smooth crossover even at $T=0$, and the system remains metallic at any $U$. It is worth not- 
ing that the last effect, the absence of an insulating phase for anomalously strong hopping disorder, is an artifact of the $d=\infty$ limit. In finite dimensions, localization and/or percolation effects are expected to restore the insulating behavior in this anomalous limit.

\subsection{Site disorder}

In addition to hopping randomness, in realistic materials one also expects some degree of site randomness, which can originate for example from the presence of long-ranged electrostatic fields due to charged impurities (acceptors). As discussed above, random site energies locally break particle-hole symmetry, even if the system is on the average at half-filling. One can thus suspect that the presence of site randomness might lead to a qualitatively different scenario, and bring into question the relevance of our result derived for models with pure hopping randomness. Motivated by these considerations, we now turn our attention to models with pure site randomness, and investigate the possible metal-insulator transitions in this case.

For simplicity, we consider a simple binary model of site randomness, where

$$
P_{S}\left(\varepsilon_{i}\right)=\frac{1}{2}\left[\delta\left(\varepsilon_{i}-\frac{1}{2} W\right)+\delta\left(\varepsilon_{i}+\frac{1}{2} W\right)\right],
$$

so that the random site energies take values $\varepsilon_{i}= \pm(1 / 2) W$, and $W$ measures the strength of disorder. In contrast to the model with uncorrelated random hopping where the disorder strength also defined the average bandwidth, this model has two independent (dimensionless) energy scales: $W / t$ and $U / t$ ( $t$ is the hopping element). For simplicity, we consider a semicircular density of states, which corresponds to the Bethe lattice in $d=\infty$.

We limit our attention to the limit of weak site disorder, and investigate the resulting behavior near the Mott transition. If we fix the disorder $W$ and increase the interactions $U$, the system undergoes the Mott transition. From the point of view of the spectral functions, this transition takes place in a fashion similar as found for hopping disorder: the coherent quasiparticle band narrows down, and eventually collapses at $U_{c}$, opening the Mott gap, corresponding to freezing one electron per site. The presence of disorder $W$ tends to broaden the bands, and thus opposes Mott freezing, so that $U_{c}(W)$ is pushed to higher values as $W$ increases.

A closer look at this transition reveals subtle differences from the hopping disorder scenario. In particular, near the Mott transition, the low energy properties (conductivity, specific heat) are determined by the structure of the coherent quasiparticle band. In the present case, the quasiparticles can be characterized by two energy scales: the renormalized disorder strength $\widetilde{W}$ and the renormalized bandwidth $\widetilde{D}$ (at $U=0$ and $W=0$ the bare bandwidth is $D=4 t$ ). Our results show that both quantities vanish at the transition. However, we found that $\widetilde{D} \sim\left(U_{\mathrm{c}}-U\right)$ (as usual) while $\widetilde{W} \sim\left(U_{\mathrm{c}}-U\right)^{2}$, so that the renormalized disorder scales to zero much faster than the renormalized bandwidth.

This phenomenon, which can be qualified as the perfect screening of the disorder by the interactions in the vicinity of the Mott transition, has important consequences for transport properties. In particular, in this limit, the scattering 
time (coming from pure site disorder) behaves as

$$
\tau_{\text {site }} \sim\left(\frac{\widetilde{D}}{\widetilde{W}}\right)^{2},
$$

and thus the conductivity

$$
\sigma_{\mathrm{DC}}(T=0) \sim \tau_{\text {site }} \rightarrow \infty
$$

as the transition is approached at which point it suddenly jumps to zero, due to the opening of the gap. Here, we have used the fact that for models with pure site disorder which are not gauge invariant, the conductivity at weak disorder can be expressed (in $d=\infty$ ) by a Drude formula, in terms of the scattering time [9]. At finite temperatures, not surprisingly, this divergence is cut off due to inelastic scattering, but at relatively low temperatures large enhancement of the conductivity still persists.

In contrast to transport properties, the thermodynamic response of the system is qualitatively the same as in the case of hopping randomness, and the specific heat coefficient (measuring the effective mass) again diverges linearly as a function of the distance from the transition.

In the above analysis, we have examined a simple model of binary site randomness. However, for more general models our conclusions about the interplay of site randomness and correlations remain valid. In particular, the existence of interaction screening of disorder persists, and the conductivity again diverges at the Mott transition. We also mention that similarly as in the case of hopping randomness, the presence of distributions of disorder with extremely long tails again leads to the suppression of the Mott insulating phase. As for hopping randomness, this effect is an artifact of the $d=\infty$ limit.

\subsection{Combined hopping and site randomness}

While we examined only the limits of pure hopping or pure site randomness so far, in most realistic systems both types will be simultaneously present. To examine this "generic" situation, we turn our attention to a model having both hopping and site randomness. For simplicity, we consider the model of hopping randomness with uncorrelated bond elements (gauge invariant models); in this case the self-consistency conditions for the local spectral functions are identical as in the case of pure site randomness on the Bethe lattice, the model that we have examined in the previous section. As a consequence, not only the local spectral functions, but also the thermodynamic behavior will be the same as discussed above.

However, the behavior of the transport properties is quite different than for pure site randomness. To see this, we recall that for the considered models of hopping randomness in addition to site randomness, the conductivity takes quite a different form than for pure site randomness. In the present case, the conductivity is expressed in terms of local spectral functions, as given in Eq. (9). As a consequence, even though the site randomness is perfectly screened by the interactions as the Mott transition is approached, the elastic scattering coming from hopping 
randomness persists, and the conductivity remains finite. More precisely, $\sigma_{\mathrm{DC}}$ approaches the value it would take in the absence of site randomness, just before the system undergoes the Mott transition and becomes insulating.

We thus conclude that the minimum metallic conductivity, i.e. the abrupt jump of the conductivity at the Mott transition is generic, and so is expected to be of relevance to experimental systems. The behavior of the models with pure site models is special, although the physics associated with the interaction screening of site randomness might be of quantitative importance even in realistic scenarios.

\section{Disordered metallic phases: the local moment formation}

Most metallic systems, both in the pure limit, and in presence of small amounts of disorder can be understood by using Fermi liquid concepts. In such situations, the excitations of the electronic system can be described as a set of weakly interacting quasiparticles, leading to universal properties at low temperatures. Of course, this simplified description is valid only at temperatures $T<T_{\text {coh }}$, where the "coherence" temperature $T_{\text {coh }}$ represents the energy scale associated with coherent quasiparticles, which measures the relative strength of the correlations in the system.

In a disordered system, the parameter that measures the relative interaction strength $u=U / t$, where $U$ is the on-site (Hubbard) interaction, and $t$ the hopping element $(t \sim$ bandwidth $)$ is also a random function of position. Those sites which are weakly hybridized with the rest of the system ( $t$ small) will be in the strong correlation regime ( $u$ large), where local charge fluctuations can be ignored, and local moment formation occurs even if the system is not very close to the Mott transition. For a broad distribution of hoppings, only few of the sites are expected to correspond to $u \gg 1$, and thus represent well formed local moments. Instead, most of the sites will be in the intermediate regime $u \sim \mathrm{O}(1)$, where the charge fluctuations cannot be ignored, and the coupling of the local moments to conduction electrons is appreciable. We expect a broad distribution of these "Kondo" couplings, leading to an even broader distribution of the corresponding "Kondo temperatures" which represent characteristic energy scales at which local Fermi liquid behavior sets in.

If the resulting distribution of the local Kondo temperatures $T_{k}$ is sufficiently broad and extends all the way to $T_{k}=0$, one can expect the behavior of the system to be qualitatively changed, and the Fermi liquid regime is not restored at any $T \neq 0$. This kind of non-Fermi liquid behavior $[19,20]$, characterized for example by a diverging specific heat coefficient $\gamma=C / T$ at $T \rightarrow 0$, is indeed observed in a number of materials containing strong hopping disorder, such as doped semiconductors. The presence of this "disorder-induced local moment formation" is also expected to play a crucial role in determining the transport properties near the metal-insulator transition.

Disorder-induced local moment formation in a Hubbard model with random hopping was recently investigated in a Hartree-Fock framework [21]. This approach does indicate the presence of instabilities to local moment formation, but it does not address the nature of local moments, or their interaction with the conduction electrons needed to determine the nature of the ground state. In 
contrast, the present approach based on the $d=\infty$ formulation offers a natural language for the problems involving the interplay of local disorder fluctuations and the strong correlation effects. Furthermore, our approach is not limited to a particular temperature interval and thus can be used to obtained results in the entire temperature range.

In order to study the physics of the disordered metallic phase, and the associated disorder-induced local moment formation, it is useful to consider models of correlated hopping randomness of the form $t_{i j}=y_{i j} x_{i} x_{j}$ introduced in Sec. 2. In realistic systems such as doped semiconductors [11], there are large fluctuations in the local hybridization of a given site with its environment originating from randomness in the position of dopant atoms. To represent such systems in our $d=\infty$ framework, it is useful to consider models with broad distributions of local hopping parameters $x_{i}$.

In general, in the case of bounded distributions of hopping elements $x_{i}$, although the local moment formation does occur, the Fermi liquid behavior is restored below a certain low temperature $T_{k}^{(\min )}$. On the other hand, if the distribution $P_{X}\left(x_{i}\right)$ extends all the way to $x_{i}=0$, the behavior of the thermodynamic and transport properties will display non-Fermi liquid aspects even at arbitrarily low temperatures. We will discuss the asymptotic low temperature behavior that can be determined analytically for an arbitrary form of $P_{X}\left(x_{i}\right)$.

\subsection{Specific heat}

We begin with the analysis of the low temperature form of the specific heat in the present case. To obtain the leading temperature dependence as $T \rightarrow 0$, we can use arguments similar to those used by Bhatt and Fisher [19], and Dobrosavljević et al. [20] in the previous work. As we have seen in Sec. 3, the energy of the system takes an additive form with respect to contributions coming from different sites. Those sites that have their respective Kondo temperatures $T_{k}(i)$ lower than the temperature of the system $T$, will provide the dominant contributions to the specific heat. To leading order, we can ignore the temperature as well as the frequency dependence of the Weiss field $W_{i}\left(\omega_{n}\right)$. In that case, the contribution coming from a given site is just that of an Anderson model with hybridization $\Delta(i)=x_{i}^{2} \Delta_{0}$, where $\Delta_{0}$ is the energy scale corresponding to the typical hybridization in the system. Since the sites in question, having very weak hybridization, act as local moments in the Kondo regime, the corresponding Kondo temperature is given by $[19,20]$

$$
T_{k}\left(x_{i}\right) \approx D_{0} \exp \left(-U / x_{i}^{2} \Delta_{0}\right)
$$

where $D_{0}$ is an energy of the order of the typical bandwidth in the system.

The contribution of a given site to $\gamma$ is then approximately given by

$$
\gamma\left(x_{i}\right) \sim \begin{cases}1 / T, & T_{k}\left(x_{i}\right)<T, \\ 1 / T_{k}, & T_{k}\left(x_{i}\right)>T .\end{cases}
$$

Collecting the contributions from all the sites with $T_{k}\left(x_{i}\right)<T$, we find that to leading order

$$
\gamma(T) \sim \frac{1}{T} n_{\mathbf{f r}}(T)
$$


Here, the number (fraction) of "free spins"

$$
n_{\mathrm{fr}}(T)=\int_{0}^{x^{\max }(T)} \mathrm{d} x_{i} P_{H}\left(x_{i}\right),
$$

and

$$
x^{\max }(T)=\left(\frac{\Delta_{0}}{U} \ln \frac{D_{0}}{T}\right)^{-2} .
$$

Using these expressions, it is not difficult to see that for any distribution $P_{H}\left(x_{i}\right)$ which extends all the way to $x_{i}=0$, and having a low $x_{i}$ tail longer than exponential (e.g. power law or lognormal), the resulting $\gamma(T)$ diverges as $T \rightarrow 0$. The precise form of this singularity depends on the details of $P_{H}\left(x_{i}\right)$. However, for any power law or even lognormal form of the low $x_{i}$ tail, the quantity $n_{\mathrm{fr}}(T)$ decreases to zero as $T \rightarrow 0$ slower than any power [20], giving an anomalously slow decrease in the number of "free" spins with temperature, and a large anomaly in $\gamma(T)$.

The above arguments rigorously shows that for a large class of models with continuous distributions of hopping our $d=\infty$ equations admit non-Fermi liquid metallic solutions.

\subsection{Conductivity}

As we have seen in the Sec. 3, the "pinning condition", which is valid for any model of hopping randomness guarantees that at particle-hole symmetry (half-filling) the $T=0$ value of the conductivity in the metallic phase remains unaffected by the interactions, and thus by the associated disorder-induced local moment formation. However, the presence of local moments does induce anomalous low-temperature corrections to $\sigma_{\mathrm{DC}}$ which could be crucial in understanding the transport properties in systems such as doped semiconductors.

To determine the leading temperature corrections to $\sigma_{\mathrm{DC}}$, we proceed in a similar fashion as in the discussion of the specific heat. In Sec. 3, we have seen that for the models under consideration, the conductivity is expressed through an averaged spectral function $\rho_{W}\left(\omega_{n}\right)$ that can be written as

$$
\rho_{W}\left(\omega_{n}\right)=\int \mathrm{d} x_{i} P_{X}\left(x_{i}\right) x_{i}^{2} \rho_{i}\left(\omega_{n}\right) .
$$

In this expression, $\rho_{i}\left(\omega_{n}\right)$ is the local spectral function (of the Anderson model) corresponding to a given site $i$, and we have assumed hopping disorder only. In the strongly correlated regime, $\rho_{i}\left(\omega_{n}\right)$ has a sharp (Kondo) peak near the Fermi surface, describing the coherent quasiparticles. As the temperature is increased from zero, inelastic scattering will destroy the existence of this coherent peak at a characteristic temperature $T_{k}$. However, in a random system, this process takes place locally, and a given site becomes "incoherent" at $T \sim T_{k}\left(x_{i}\right)$, when this Kondo resonance is washed out. Thus, in contrast to thermodynamic response, appreciable contributions to the conductivity come from those sites with $T_{k}\left(x_{i}\right)>T$, which remain coherent. More precisely,

$$
\rho_{i}\left(\omega_{n} \rightarrow 0\right) \sim \begin{cases}0, & T_{k}\left(x_{i}\right)<T, \\ 1 / x_{i}^{2} \Delta_{0}, & T_{k}\left(x_{i}\right)>T .\end{cases}
$$


Again, to leading order we can ignore the frequency dependence of $\rho_{i}\left(\omega_{n}\right)$, and we find $\left(\right.$ at $\left.\omega_{n} \rightarrow 0\right)$

$$
\rho_{W}(T) \sim \int_{x_{\max }(T)}^{+\infty} \mathrm{d} x_{i} P_{X}\left(x_{i}\right) .
$$

By using this result, and Eq. (11), we find that the leading low temperature correction to the $\mathrm{DC}$ conductivity assumes the form

$$
\delta \sigma_{\mathrm{DC}}(T) \sim n_{\mathrm{fr}}(T) .
$$

It is interesting to note that, although $n_{\mathrm{fr}}(T)$ vanishes slower than any power as $T \rightarrow 0$, for realistic distributions one can write

$$
n_{\mathrm{fr}}(T)=T^{\alpha(T)} \text {, }
$$

where $\alpha(T)$ only very weakly, typically logarithmically [20], depends on temperature. Experimentally, one expects to measure some effective exponent $\alpha$. As $\alpha \rightarrow 0$ at $T \rightarrow 0$, one expects these effective exponents to be small. This behavior is to be contrasted with the fact that similar, nonanalytic finite temperature corrections to the conductivity of dirty metals follow from weak localization and interaction [12] effects. Our results thus suggest that such temperature dependence, could have an entirely different origin - due to disorder-induced local moment formation in a strongly correlated metal. Of course, a full theory should include both the local moments and the mentioned hydrodynamic (diffusion) corrections. In the framework of our approach these additional terms would appear at the level of one-loop corrections to the $d=\infty$ (mean-field) expressions.

\section{Functional integral formulation and loop expansion}

The functional integral representation of the (replicated) partition function of the model can be written as

$$
\overline{Z^{n}}=\int D \varepsilon_{i} P \varepsilon_{i} D t_{i j} P t_{i j} \int D \bar{c}_{i} D c_{i} \exp (-S),
$$

with the action $S=S_{\text {loc }}+S_{\text {hop }}$ consisting of a local part (that includes the Hubbard interaction)

$$
\begin{aligned}
& S_{\mathrm{loc}}=\sum_{i} S_{l o c}(i)= \\
& =\sum_{i}\left[\sum_{\alpha, s} \int_{0}^{\beta} \mathrm{d} \tau \bar{c}_{s, i}^{\alpha}\left(\partial_{\tau}+\varepsilon_{i}-\mu\right) c_{s, i}^{\alpha}+U \sum_{a} \int_{0}^{\beta} \mathrm{d} \tau \bar{c}_{\uparrow, i}^{\alpha} c_{\uparrow, i}^{\alpha} \bar{c}_{\downarrow, i}^{\alpha} c_{\downarrow, i}^{\alpha}\right],
\end{aligned}
$$

and a hopping part

$$
S_{\text {hop }}=\sum_{\langle i j\rangle} S_{\text {hop }}(i, j)=\sum_{\langle i j\rangle}\left[t_{i j} \sum_{\alpha, s} \int_{0}^{\beta} \mathrm{d} \tau\left(\bar{c}_{s, i}^{\alpha} c_{s, j}^{\alpha}+\text { h.c. }\right)\right] .
$$

Here, $\bar{c}_{s, i}^{\alpha}$ and $c_{s, i}^{\alpha}$ are the electronic (Grassmann) fields with spin $s=\uparrow, \downarrow$, replica index $\alpha=1, \ldots, n$, at lattice site $i$, and $\beta$ is the inverse temperature. The random site energies $\varepsilon_{i}$ are described by their probability distribution $P_{S} \varepsilon_{i}$, and the random hopping elements $t_{i j}$ by the corresponding distribution $P_{H} t_{i j}$. 
At this point, it is convenient to perform explicitly the averaging over the Gaussian random (bond) variables $y_{i j}$, after which the hopping part of the action takes the form

$$
S_{\text {hop }}=\frac{1}{2} t^{2} \sum_{i j} \frac{1}{z} f_{i j} x_{i}^{2} x_{j}^{2}\left[\sum_{\alpha, s} \int_{0}^{\beta} \mathrm{d} \tau\left[\bar{c}_{s, i}^{\alpha}(\tau) c_{s, j}^{\alpha}(\tau)+\text { h.c. }\right]\right]^{2} .
$$

As we can see from this expression, the averaging over disorder has generated a quartic term in the action, that is nonlocal in (imaginary) time, spin and replica indices. We are now in a position to introduce a collective $Q$-fields of the form (in terms of Matsubara frequencies $\omega=2 n \pi T$; the indices "' are omitted for brevity)

$$
Q_{\omega_{1} \omega_{2}}^{\alpha_{1} \alpha_{2}, s_{1} s_{2}}(i)=\frac{1}{z} \sum_{j} f_{i j} x_{j}^{2} \bar{c}_{j, s_{1}}^{\alpha_{1}}\left(\omega_{1}\right) c_{j, s_{2}}^{\alpha_{2}}\left(\omega_{2}\right)
$$

by decoupling the (quartic) hopping term using a Hubbard-Stratonovich transformation. For simplicity, as before, we will ignore the superconducting phases, as well as the fluctuations in the particle-particle (Cooper) channel, so that the $Q$-field does not have anomalous components. The procedure can be straightforwardly generalized to include the omitted terms.

It is now possible to integrate out formally the electron (Grassmann) fields, and the resulting action for the $Q$-fields can be written as

$$
S Q=S_{\mathrm{hop}} Q+S_{\mathrm{loc}} Q .
$$

The nonlocal part of the action $S_{\text {hop }} Q$ takes a simple quadratic form in terms of the $Q$ fields

$$
S_{\mathrm{hop}} Q=-\frac{1}{2} t^{2} \sum_{i j} \sum_{\alpha_{1} \alpha_{2}} \sum_{s_{1} s_{2}} \sum_{\omega_{1} \omega_{2}} K_{i j} Q_{\omega_{1} \omega_{2}}^{\alpha_{1} \alpha_{2}, s_{1} s_{2}}(i) Q_{\omega_{2} \omega_{1}}^{\alpha_{2} \alpha_{1}, s_{2} s_{1}}(j)
$$

where, $K_{i j}=(1 / z) f_{i j}^{-1}$ is the inverse lattice matrix, scaled by a coordination number $z$. In contrast, all the nonlinearities are contained in the local part of the action

$$
\begin{aligned}
& S_{\mathrm{loc} Q}=-\sum_{i} \ln \int \mathrm{d} x_{i} P_{X}\left(x_{i}\right) \int \mathrm{d} \varepsilon_{i} P_{S}\left(\varepsilon_{i}\right) \int D \bar{c}_{i} D c_{i} \\
& \quad \times \exp \left(-S_{\mathrm{eff}} \bar{c}_{i}, c_{i}, Q_{i}, x_{i}, \varepsilon_{i}\right),
\end{aligned}
$$

where the effective action for on-site electrons takes the form

$$
\begin{aligned}
& S_{\mathrm{eff}} \bar{c}_{i}, c_{i}, Q_{i}, x_{i}, \varepsilon_{i}=-\sum_{\alpha_{1} \alpha_{2}} \sum_{s_{1} s_{2}} \sum_{\omega_{1} \omega_{2}} \bar{c}_{i, s_{1}}^{\alpha_{1}}\left(\omega_{1}\right)\left(\mathrm{i} \omega_{1}+\mu-\varepsilon_{i}\right) \delta_{\alpha_{1} \alpha_{2}} \delta_{s_{1} s_{2}} \delta_{\omega_{1} \omega_{2}} \\
& \quad-x_{i}^{2} t^{2} Q_{\omega_{1} \omega_{2}}^{\alpha_{1} \alpha_{2}, s_{1} s_{2}}(i) c_{i, s_{2}}^{\alpha_{2}}\left(\omega_{2}\right) \\
& \quad+U \sum_{\alpha} \sum_{\omega_{1}+\omega_{3}=\omega_{2}+\omega_{4}} \bar{c}_{i, \uparrow}^{\alpha}\left(\omega_{1}\right) c_{i, \uparrow}^{\alpha}\left(\omega_{2}\right) \bar{c}_{i, \downarrow}^{\alpha}\left(\omega_{3}\right) c_{i, \downarrow}^{\alpha}\left(\omega_{4}\right) .
\end{aligned}
$$

The local effective action $S_{\text {eff }} \bar{c}_{i}, c_{i}, Q_{i}, x_{i}, \varepsilon_{i}$ is identical to the action of a (generalized) Anderson impurity model embedded in a electronic bath characterized by a hybridization function $x_{i}^{2} t^{2} Q_{\omega_{1} \omega_{2}}^{\alpha_{1} \alpha_{2}, s_{1} s_{2}}(i)$. We can thus interpret our system as a collection of Anderson impurity models that are "connected" through the existence of a collective $Q$-fields. Here we note that, in contrast to an ordinary Anderson model, the hybridization function is now non-diagonal in frequency, spin 
and replica indices. Physically, this reflects the fact that in general dimensions a given site can be regarded as an Anderson impurity model in a fuctuating bath, which breaks local translational invariance in time, space and spin.

When $z \rightarrow \infty$, the functional integral over $Q$-fields, representing the partition function, can be evaluated (exactly) by a saddle-point method, and we obtain a mean-field theory. In order to derive the mean-field equations in our case, we look for extrema of the action $S Q$ with respect to the variations of the $Q$-fields, i.e.

$$
\frac{\delta S Q}{\delta Q_{\omega_{1} \omega_{2}}^{\alpha_{1} \alpha_{2}, s_{1} s_{2}}(i)}=0
$$

The saddle-point solution is translationally invariant in time, space, and conserves spin, and is diagonal in all indices

$$
\left[Q_{\omega_{1} \omega_{2}}^{\alpha_{1} \alpha_{2}, s_{1} s_{2}}(i)\right]_{\mathrm{SP}}=\delta_{\alpha_{1} \alpha_{2}} \delta_{s_{1} s_{2}} \delta_{\omega_{1} \omega_{2}} Q_{s}^{\mathrm{SP}}(\omega)
$$

and the saddle-point equations assume the form

$$
Q_{s}^{\mathrm{SP}}(\omega)=\int \mathrm{d} \varepsilon_{i} P_{S}\left(\varepsilon_{i}\right) \int \mathrm{d} x_{i} P_{X}\left(x_{i}\right) x_{i}^{2} G_{i, s}(\omega),
$$

where

$$
G_{i, s}(\omega)=\left\langle\bar{c}_{s}(\omega) c_{s}(\omega)\right\rangle_{S_{\mathrm{efr}} \bar{c}, c, Q^{\mathrm{SP}, x_{i}, \varepsilon_{i}}} .
$$

If we identify

$$
W_{s, i}(\omega) \equiv x_{i}^{2} t^{2} Q_{s}^{\mathrm{SP}}(\omega)
$$

we obtain the mean-field equations presented in Sec. 2 .

The present approach is particularly convenient for the study of the effects of strong correlations on disorder-driven transitions, and the interplay of Anderson localization and strong correlations in general. This is especially true, since Anderson localization is not present in $d=\infty$ (or infinite range) models, and so one has to extend the approach to include the presence of spatial fluctuations missing from the mean-field description. In order to study systematically the fluctuation effects, we proceed to carry out an expansion in terms of the deviations of the collective $Q$-fields from their saddle-point value, i.e. in powers of $\delta Q(i)=Q(i)-Q^{\mathrm{SP}}$. This procedure, also known as a loop expansion has been used in other disordered problems, such as spin-glasses, to generate systematic corrections to the mean-field theory. The method is particularly convenient when applied to long-range models (class (b) above), since in that case the loop corrections are ordered by a small parameter $1 / z$. The loop expansion can be applied also to large dimensionality models (class (a) above), but in that case a given order in a loop expansion can be considered to be an infinite resummation of the simple $1 / d$ expansion, since each term contains all powers of $1 / d$.

When the expansion of the effective action in terms of $\delta Q$ is carried to the lowest, quadratic order, we obtain a theory describing Gaussian fluctuations around the saddle point, that represent weakly interacting collective modes. Higher order terms in the expansion then generate effective interactions of these modes, which under appropriate conditions can lead to fluctuation-driven phase transitions.

The Gaussian fluctuations of the $Q$-fields, will allow us to compute the leading corrections to the mean-field theory. They have the form 


$$
\begin{gathered}
S^{(2)}[Q]=-\frac{1}{2} t^{2} \sum_{l_{1} \cdots l_{4}} \int \frac{\mathrm{d} k}{(2 \pi)^{d}} \delta Q_{l_{1} l_{2}}(k)\left[\left(L^{2} k^{2}+1\right) \delta_{l_{1} l_{4}} \delta_{l_{2} l_{3}}\right. \\
\left.-t^{2} W\left(l_{1}\right) W\left(l_{3}\right) \delta_{l_{1} l_{2}} \delta_{l_{3} l_{4}}+t^{2} \Gamma\left(l_{1} \cdots l_{4}\right)\right] \delta Q_{l_{3} l_{4}}(-k) .
\end{gathered}
$$

This expression is appropriate for the long ranged model (b) above, in which case the inverse lattice matrix in momentum space takes the form $K(k) \approx 1+L^{2} k^{2}$, and we cut off the momentum integrals at $A=2 \pi / L$. Note that the coefficient of $k^{2}$, which can be interpreted as stiffness of the $\delta Q$ modes, is $\sim L^{2}$, so we see that indeed the fluctuations are suppressed at $l \rightarrow \infty$. In the above formula, the index $l_{m}$ is used to represent the frequency, spin and replica indices. The local vertex function $\Gamma\left(l_{1} \cdots l_{4}\right)$ is given by

$$
\Gamma\left(l_{1} \cdots l_{4}\right)=\int \mathrm{d} \varepsilon_{i} P_{S}\left(\varepsilon_{i}\right) \int \mathrm{d} x_{i} P_{X}\left(x_{i}\right) x_{i}^{4}\left\langle\bar{c}_{i}\left(l_{1}\right) c_{i}\left(l_{2}\right) \bar{c}_{i}\left(l_{3}\right) c_{i}\left(l_{4}\right)\right\rangle_{S_{\mathrm{et} 1} Q^{\mathrm{sP}}}
$$

At this level, the dynamics of the collective fluctuations $\delta Q$ is governed by the form of $S^{(2)} \delta Q$, which is expressed in terms of the local correlation functions of the saddle-point theory, i.e. of the $d=\infty$ disordered Hubbard model. Accordingly, a detailed study of the $d=\infty$ limit does not provide only a mean-field description of the problem, but also determines the form of the leading corrections resulting from fluctuations.

\section{Comparison with experiments}

After three decades of intensive investigations, there are various constraints on a theoretical model which purports to explain the physics of uncompensated doped semiconductors. In the following, we list these constraints and comment how our perspective fares vis a vis the experiments.

(1) At the accessible temperatures, the thermodynamic quantities $\chi$ and $\gamma$ vary smoothly [11] as a function of concentration across the transition, and are increasing functions of the inverse temperature even throughout the metallic phase.

(2) The thermodynamic behavior should be contrasted with the measurements of charge transport, which vary much more rapidly near the transition. The $T=0$ extrapolated value [11] of the conductivity vanishes with an exponent $\mu$ which is thought to be close to $1 / 2$. However, no dynamical scaling range in temperature and concentration has been observed, making the determination of the exponents ambiguous. In particular, values of the conductivity exponent ranging from $\mu=0$ [23] to $\mu=1$ [22] have been obtained on the basis of the same data.

(3) The NMR experiments [24] portray a strongly inhomogeneous picture. The Knight shift on phosphorus is much larger than that on Si. Furthermore, the $1 / T_{1} T$ on $\mathrm{Si}$ is the temperature dependence of $\chi^{2 *}$. This can be understood naturally if the magnetic susceptibility has a weak momentum dependence.

(4) Finkelshtein has emphasized [25] that the ESR line width [26] $\Delta H_{1 / 2}$ is proportional to $\chi$. Since $\Delta H_{1 / 2}$ is proportional to $\sum_{q} \chi(q)$, this indicates that the divergent part of the susceptibility is roughly $q$ independent.

(5) Small amount of compensation or the external magnetic field are very relevant perturbations [11] that dramatically alter the critical behavior of the conductivity.

\footnotetext{
*This observation is due to A. Georges.
} 
(6) Spin-orbit scattering however is an irrelevant perturbation [27] since Si:B (where spin-orbit scattering is dominant) shows similar behavior as Si:P.

The approach presented in this paper, on the most qualitative level, is consistent with all these observations on the metallic side.

The collection of Anderson impurity models as a mean-field theory of the disordered Hubbard model provides a microscopic realization of the two-fluid model which phenomenologically explains (1).

Underlying this picture there is a broad distribution of energy scales which makes the Mott transition in dirty systems very different than in the pure case. The conductivity goes discontinuously to zero at $T=0$, but at any finite temperature is a rapidly varying continuous function. This observation may account for the lack of scaling, but here the loop corrections to the mean-field theory might modify the effective exponents.

The local inhomogeneity of the system described in (3) is naturally captured in our formulation. The weakly coupled sites can be thought of as the $\mathrm{P}$ donors where the wave function of the delocalized electrons are concentrated.

The $q$ independence of the thermodynamic response discussed in (4) is directly built in our formulation at the mean-field level. The loop expansion corrections will bring possible departures from this behavior.

In our model, the relevant perturbations are those that cause departures from particle-hole symmetry. Our approach thus justifies the fact that the uncompensated doped semiconductors represent a unique universality class.

\section{Conclusions}

We have presented some new results of a nonperturbative approach to strongly correlated disordered electrons. Even at the mean-field level, our results agree with most of the surprising features found in doped semiconductors. Furthermore, our approach allows systematic corrections to be obtained beyond the mean-field theory. In this way, it will be possible to incorporate the effects of long-wavelength (hydrodynamic) fluctuations responsible for the weak-localization and interaction corrections and to make contact with the existing scaling theories of localization.

\section{Acknowledgments}

We thank E. Abrahams, R. Bhatt, A. Ruckenstein, M. Sarachik, and Q. Si for useful discussions. This work was supported by the NSF under grant DMR 92-24000 and the ONR under grant N-11378.

\section{References}

[1] N.F. Mott, Phil. Mag. 6, 287 (1961).

[2] S.A. Carter, T.F. Rosenbaum, J.M. Honig, J. Spałek, Phys. Rev. Lett. 67, 3440 (1991).

[3] J. Hubbard, Proc. R. Soc. Lond. A, Math: Phys. Sci. 281, 401 (1964).

[4] W.F. Brinkman, T.M. Rice, Phys. Rev. B 2, 4302 (1970). 
[5] J.C. Slater, Phys. Rev. 82, 538 (1951).

[6] W. Metzner, D. Vollhardt, Phys. Rev. Lett. 62, 324 (1989).

[7] A. Georges, G. Kotliar, Phys. Rev. B 45, 6479 (1992).

[8] D. Belitz, T.R. Kirkpatrick, Phys. Rev. Lett. 63, 1296 (1989); Physica A 167, 259 (1990).

[9] V. Dobrosavljević, G. Kotliar, Phys. Rev. Lett. 71, 3218 (1993).

[10] For related work on this subject see V. Janis, M. Ulmke, D. Vollhardt, preprint (1993); G.S. Uhrig, R. Vlaming, preprint (1993).

[11] For a recent review of doped semiconductors, see M.A. Paalanen, R.N. Bhatt, Physica B 169, 231 (1991).

[12] For a review of disordered electronic systems see P.A. Lee, T.V. Ramakrishnan, Rev. Mod. Phys. 57, 287 (1985).

[13] A.M. Finkelshtein, Zh. Eksp. Teor. Fiz. 84, 168 (1983); Sov. Phys. JETP 57, 97 (1983); 86, 367 (1984); 59, 212 (1983); Z. Phys. B 56, 189 (1984).

[14] The Falicov-Kimball model with random-site energies in $d=\infty$ was recently examined by V. Janis, D. Vollhardt, Phys. Rev. B 46, 15712 (1992).

[15] F. Wegner, Z. Phys. B 25, 327 (1976); L. Schäffer, F. Wegner, ibid. 38, 113 (1980).

[16] Th. Pruschke, d. L. Cox, M. Jarrel, Europhys. Lett. 21, 5 (1993); Phys. Rev., to be published.

[17] A. Khurana, Phys. Rev. Lett. 64, 1990 (1990).

[18] M. Jarrel, Phys. Rev. Lett. 69, 168 (1992); M.J. Rozenberg, X.Y. Zhang, G. Kotliar, Phys. Rev. Lett. 69, 1236 (1992); X.Y. Zhang, M.J. Rozenberg, G. Kotliar, Phys. Rev. Lett. 70, 1666 (1993).

[19] R.N. Bhatt, D.S. Fisher, Phys. Rev. Lett. 68, 3072 (1992).

[20] V. Dobrosavljević, T.R. Kirkpatrick, G. Kotliar, Phys. Rev. Lett. 69, 1113 (1992).

[21] M. Milovanović, S. Sachdev, R.N. Bhatt, Phys. Rev. Lett. 63, 82 (1989).

[22] H. Stupp, M. Hornung, M. Lakner, O. Madel, H. Lóhneysen, unpublished.

[23] A. Möbius, Z. Phys. B 80, 213 (1990); Phys. Rev. B 40, 4194 (1989).

[24] M.A. Paalanen, A.E. Ruckenstein, G.A. Thomas, Phys. Rev. Lett. 54, 1295 (1985); H. Alloul, P. Dellouve, Phys. Rev. Lett. 59, 578 (1987).

[25] A.M. Finkelshtein, Pis'ma Zh. Eksp. Teor. Fiz. 46, 407 (1987).

[26] M.A. Paalanen, S. Sachdev, R. Bhatt, A.E. Ruckenstein, Phys. Rev. Lett. 57, 2061 (1986).

[27] P. Dai, Y. Zhang, M.P. Sarachik, Phys. Rev. Lett. 66, 1941 (1991); Phys. Rev. B 45, 3984 (1992). 